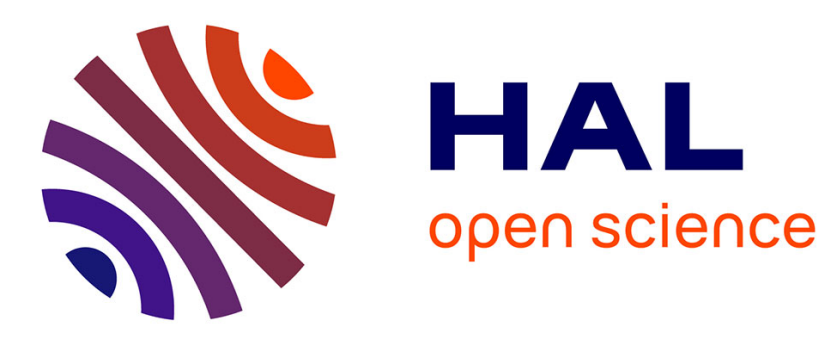

\title{
Towards Multi-instances QoS Efficient RPL for Smart Grids
}

\author{
Jad Nassar, Nicolas Gouvy, Nathalie Mitton
}

\section{To cite this version:}

Jad Nassar, Nicolas Gouvy, Nathalie Mitton. Towards Multi-instances QoS Efficient RPL for Smart Grids. PE-WASUN 2017 - 14th ACM International Symposium on Performance Evaluation of Wireless Ad Hoc, Sensor, and Ubiquitous Networks, Nov 2017, Miami, FL, United States. pp.85-92, 10.1145/3134829.3134838. hal-01611062

\section{HAL Id: hal-01611062 \\ https://hal.science/hal-01611062}

Submitted on 7 Dec 2017

HAL is a multi-disciplinary open access archive for the deposit and dissemination of scientific research documents, whether they are published or not. The documents may come from teaching and research institutions in France or abroad, or from public or private research centers.
L'archive ouverte pluridisciplinaire HAL, est destinée au dépôt et à la diffusion de documents scientifiques de niveau recherche, publiés ou non, émanant des établissements d'enseignement et de recherche français ou étrangers, des laboratoires publics ou privés. 


\section{Towards Multi-instances QoS Efficient RPL for Smart Grids}

\author{
Jad Nassar \\ HEI - Yncrea HdF, France \\ Inria, France \\ Jad.nassar@\{yncrea,inria\}.fr
}

\author{
Nicolas Gouvy \\ HEI - Yncrea HdF, France \\ Nicolas.gouvy@yncrea.fr
}

\author{
Nathalie Mitton \\ Inria, France \\ Nathalie.mitton@inria.fr
}

\begin{abstract}
The Internet of Thing is an ongoing revolution which promises to interconnect most of our world with billions of connected devices. Hence, data routing and prioritization in IoT is a main challenge in this gigantic network. This is all the more true for the Smart Grids data management where heterogeneous applications and signalling messages have different requirements in terms of reliability, latency and priority. So far, standards on Smart Grid have recommended the use of RPL (Routing Protocol for Low-Power and Lossy networks) protocol for distributing commands over the grid. RPL assures Quality of Service (QoS) at the network layer in wireless sensor networks through the logical subdivision of the network in multiple instances, each one relying on a specific Objective Function. However, RPL is not optimized for Smart Grids, as its main objective function and its associated metric does not allow for QoS differentiation. In order to overcome this, we propose $O F Q S$ an objective function with a multi-objective metric that considers the delay and the remaining energy in the battery nodes alongside with the quality of the links. Our function automatically adapts to the number of instances (traffic classes) providing a QoS differentiation based on the different Smart Grid applications requirements. Simulations show that our proposal provides a low packet delivery latency and a higher packet delivery ratio while extending the lifetime of the network compared to literature solutions.
\end{abstract}

\section{KEYWORDS}

Smart Grid; RPL; routing; QoS; objective function; metric.

\section{INTRODUCTION}

Current electric grid no longer satisfies the energy needs of the twenty first century. The increased electricity offer per person is limited by the restrained electricity production and the aging and unsuitable infrastructures. This limitation is due to inaccurate management systems, inefficient operations and maintenance processes and the centralized communication system that lacks interoperability. Besides that, the introduction into the electricity grid of multiple sporadic Distributed Energy Resources (DERs) i.e electric vehicles, photovoltaic cells, wind farms, located in sometimes unexpected places, makes the control of it even more complicated [5] . Smart Grid (SG) projects, such as the SoMel SoConnected project $^{1}$, promises to solve these issues by operating with automatic control and operation in response to user needs and power availability improving efficiency, reliability and safety, with smooth integration of renewable and alternative energy sources. Managing the SG with a ubiquitous network to exchange regular and critical control messages all-over the power network becomes then crucial. Based on these observations and in order to shift from the existing electric grid to the SG, it appears necessary to instrument and master the high and complex energy management on the electric grid. Consequently, one of the potential solutions envisioned is to equip the electrical grid with wireless sensors located at strategic measuring points to achieve remote monitoring, data collection and control of the grid [16]. Simultaneously, such sensors will constitute a parallel wireless data network to the electrical grid. SG applications are heterogeneous in terms of requirements, criticality and delay tolerance [4] [9] [24] (Table 2 shows the delay tolerance and reliability levels for the different SG applications).However, since these applications will generate different types of traffic (real-time, critical, regular) [25], they will require different levels of QoS. Thus, for a wireless sensor network, different criteria have to be taken into consideration in order to achieve a proper communication with the following requirements: reliability, latency, auto-configuration, auto-adaptation, network scaling and data prioritization [25]. Among all the existing routing protocols used in the SGs, the IEEE standard RPL [29] remains the most recognized and widely used [28] [2] [23]. As a general protocol, RPL is intended to meet the requirements of a wide range of Low-Power and Lossy Networks (LLNs) application domains including the SGs ones. It provides different QoS classes at the network layer through multiple logical subdivisions of the network called instances (more details in section 2). Following RPL, an IETF draft [7] proposes five different priority classes for the traffic in SG AMI. Other papers classify the traffic into two levels of critical and periodic [6]. Based on that and since the traffic classes in the SG are not standardized, a single solution to route the traffic with different QoS may not be sufficient since the number of instances (traffic classes) vary depending on the application and the implementation. A multi-objective solution is then essential to meet the QoS requirements and achieve a reliable communication in the SG. Therefore, in this paper, we introduce $O F Q S$ an RPL-compliant objective function, with a multi-objective metric that considers the delay and the remaining energy in the battery nodes alongside with the quality of the links. Our function automatically adapts to the number of instances (traffic classes) providing a QoS differentiation based on the different Smart Grid applications requirements. Simulations show that $O F Q S$ provides a low packet delivery latency and a higher packet delivery

\footnotetext{
${ }^{1}$ http://livetree.fr/
} 
ratio while extending the lifetime of the network compared to literature solutions.

The rest of the paper is organized as follows: Section 2 provides a brief overview about the RPL protocol. Section 3 presents prior works around the RPL protocol concerning the metrics and the multiple instances. Section 4 describes our proposition in details. Section 5 shows the simulation parameters and environment used to validate our proposition. Section 6 presents the performance evaluation of our proposition. Finally, Section 7 concludes the paper.

\section{RPL PROTOCOL OVERVIEW}

RPL is a Distance Vector based IPv6 routing protocol for LLNs. It divides the network into multiple logical graphs called DODAGs. DODAGs are tree-like structures built in order to avoid loops. Each node of a DODAG has a rank (distance from the root), and this rank must increase by going down the tree from the root. RPL can use multiple overlapping DODAGs over the entire network to provide different levels of QoS in the network layer. In this case, each level/DODAG is called an instance. Thus an RPL network contains at least one instance. And an instance consists itself of one or more DODAGs. Hence, a node can join a single DODAG per instance, but it can participate in multiple instances to carry different types of traffic simultaneously. An RPL instance is associated with an objective function in order to optimize the topology based on several metrics/constraints such as the shortest path or the quality of the links.

MRHOF [15] and OFO [26] are the two standardized functions in RPL. MRHOF uses ETX metric by default. OFO uses the "step_of_rank" to compute the amount by which to increase the rank along a particular link using static (Hop count) or dynamic metrics (ETX). Whichever the metric, a DODAG construction starts from the root by sending DODAG Information Object (DIO) messages to its neighbors. The DIO contains the metric/constraint used by the objective function and the rules to join a DODAG. Nodes will receive and process DIO messages potentially from multiple nodes and make a decision according to the objective function and local policies (if existing) whether to join the graph or not. Once a node joins a graph it has a route towards the parent node and the rest of the graph towards to root. The node then computes the rank of itself within the graph, which indicates the coordinates of the node in the graph hierarchy. If configured to act as a router, it starts advertising the graph information with the new information to its own neighboring nodes. If the node is a leaf node, it simply joins the graph and does not send any DIO message. The neighboring nodes will repeat this process and do parent selection, route addition and graph information advertisement using DIO messages. At the end of this process only upward routes (i.e to the root) are built. To establish downward routes a node must send a Destination Advertisement Object (DAO) to its parent containing prefix information of the nodes in its sub-DODAG, when the message arrives to the root, the prefixes are aggregated and the downward routes are then built and available to the parents. RPL nodes can also send DODAG Information Solicitation (DIS) messages to solicit DIO messages from neighbors. RPL uses the trickle algorithm to optimize DIO messages rate. For example, if the number of DIO messages sent within an interval is not consistent with the network state, the RPL resets the trickle timer to a minimum value. Otherwise, if the number of DIO messages is bigger than a certain threshold the trickle interval (DIO message rate sending) is doubled up to a maximum value "DIO_INTERVAL_MAX".

\section{RELATED WORKS}

Many researches are active around RPL in order to adapt it to different Internet of Things applications. Moreover many critical analysis were made to highlight the gaps concerning the reliability and used metrics in the aforementioned protocol in a SG environment [1] [2] [20].

In [27] several routing metrics were proposed to be used for path calculation in LLN, i.e the Throughput, Node Energy, Latency, Link reliability with the LQL (Link Quality Level) or ETX metric. ETX in MRHOF [15] and HC (Hop Count) in $O F 0$ [26] are the two main metrics used in the OFs. ETX finds paths with the fewest expected number of transmissions (including retransmissions) required to deliver a packet all the way to its destination [11]. Although $\boldsymbol{E T X}$ is reliable and widely used as a metric in wireless sensor networks, it does not take into account directly the latency which is critical in some SG applications [7]. And $\boldsymbol{E T X}$ is not energy aware, thus for a link with few re-transmissions, ETX will keep sending packets on it without taking the decrease of battery nodes level into account. $\boldsymbol{H C}$ only takes the number of hops into consideration to calculate the best path which is not always satisfactory in LLN.

An energy-based objective function for RPL that uses the remaining energy as the main routing metric was proposed in [17]. It achieves a better load balancing and increases the network lifetime comparing to $\boldsymbol{E T X}$ but with a lower delivery ratio.

In [10] two MAC aware routing metrics were proposed to be used in RPL: R-metric and Q-metric. R-metric extends $\boldsymbol{E T X}$ by considering packet losses due to the MAC contention. Q-metric provides load balancing by selecting the lightest parent in terms of traffic load by solving an optimization problem and mainly considering reliability, transmission and reception power consumption. ETT-LB was proposed in [30]. It is based on the ETT metric [12], which extends $\boldsymbol{E T X}$ by considering the link transmission rate and packet size, adding to it the Expected Delay Time (EDT), which is the average link load at a node in order to achieve load balancing. In [8] $L^{2} A M$ metric was proposed. It is based on an combination of both data reliability (defined by $\boldsymbol{E T X}$ ) and the nodes residual energy. Although their solution extended the network lifetime, it remains not adapted to a network with heterogeneous applications in terms of criticality and powered/battery nodes. Metric combination was also considered in several works. Fuzzy logic metric combination was proposed in [14] and [18] 
in order to be used for RPL. They combined several metrics like end-to-end delay, $\boldsymbol{H C}$, link quality and battery level. In [19] two combinations of two metrics were proposed: lexical and additive. In the lexical combination, the second metric is inspected only if the first one leads to equal paths, while in the additive combination the paths are calculated based on a different cost given to each metric. Multiple instances in RPL and QoS were studied in many works [23] [21] [3]. Yet, these works limit the number of instances to two and don't take into consideration the drawbacks of the used metrics (ETX and $\boldsymbol{H C}$ ) concerning the energy efficiency and end-to-end delay.

As a conclusion, a single routing metric cannot assure traffic differentiation in a SG since different applications require different QoS levels. In addition, in a multiple instance environment, the chosen objective function/metric has to guarantee the QoS requirements of the concerned SG application, which to the best of our knowledge has not been proposed yet. This is why we propose $O F Q S$ with its multiobjective metric $\mathbf{m O F Q S}$ taking account of these requirements and improving the communication in the SG. Finally, note that $O F Q S$, by integrating the different requirements of the SG applications, is suitable for any other application with these same demands and criticality variations i.e Smart City applications.

\section{PROPOSED SOLUTION}

\subsection{OFQS Objective function}

$O F Q S$ is intended to be used with the tunable multi-objective metric $\mathbf{M O F}$ QS. This metric adapts automatically to the number of instances in the network depending on their criticality level by tuning its parameters dependently. OFQS is derived from $M R H O F$ as it relies on the same rank calculation mechanism, it adopts hysteresis to prevent routing instabilities and reduces parent switches under a certain threshold.

\subsection{QoS factors in $O F Q S$}

$O F Q S$ with its metric $\mathbf{m O F Q S}$ takes the quality of the links into consideration by calculating their $\boldsymbol{E T X}$ value. In Contiki Operating System [13], ETX is implemented in the MRHOF objective function. $\boldsymbol{E T X}$ is updated based on callbacks from the MAC layer which gives information whether a MAC layer transmission succeeded, and how many attempts were required. Smaller ETX values mean better links quality to route the packets with less re-transmissions. Alongside with the quality of links, the delay is an important factor as already mentioned in SG applications. For that, $\mathbf{m O F Q S}$ considers the delay of time $d$ between sending the packet and receiving it in the network layer between two nodes. This will allow the algorithm to choose faster links specially for critical applications considering both queuing and interference delays. Moreover, in a SG, electricity and energy do exist, but connecting sensors to such high voltage with intermittent and ill-adapted energy levels is sometimes inappropriate. For that, battery-powered sensors must be deployed all over the grid alongside with the main-powered ones. Different requirements for different applications may tolerate in some cases passing by a longer route in order to preserve the remaining energy in the nodes. Hence, considering the battery level for the nodes in our metric will be beneficial in terms of traffic load balancing and network lifetime. In order to do so, we classify the remaining energy in the nodes into three Power States [22]:

- $P S=3$ : Full battery state (ranging between $100 \%$ and $80 \%$ ) or main powered

- $P S=2$ : Normal battery state (ranging between $80 \%$ and $30 \%)$

- $P S=1$ : Critical battery state (less then $30 \%$ )

By using this classification, weak nodes become unfavorable in the route selection by penalizing the ones with a smaller $P S$. We note that these levels could be adjusted for other applications depending on the network characteristics.

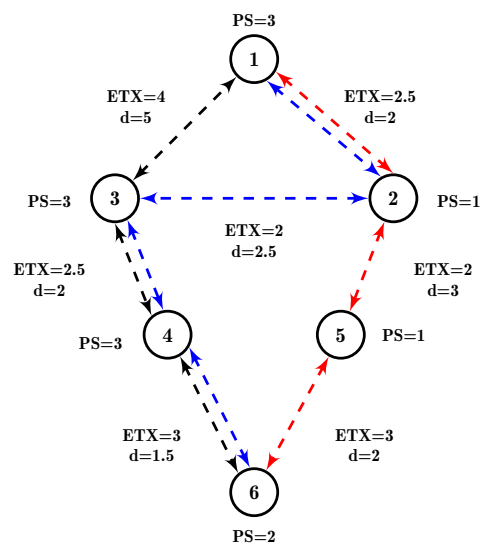

Figure 1: Network with different ETX, delay $d$ (in ms) and $P S$ values

\section{Paths}

\begin{tabular}{|c|c|c|c|}
\hline Metrics & $\begin{array}{l}\text { Path } 1 \\
6->5->2->1\end{array}$ & $\begin{array}{l}\text { Path } 2 \\
6->4->3->1 \\
\end{array}$ & $\begin{array}{l}\text { Path } 3 \\
6->4->3->2->1 \\
\end{array}$ \\
\hline $\begin{array}{l}\text { Instance } 1 \\
\boldsymbol{E T X}\end{array}$ & 7.5 & 9.5 & 10 \\
\hline Instance 2 & - & - & - \\
\hline $\begin{array}{l}\text { Instance } 1 \\
\boldsymbol{E T X}\end{array}$ & 7.5 & 9.5 & 10 \\
\hline $\begin{array}{l}\text { Instance } 2 \\
\mathbf{H C}\end{array}$ & 3 & 3 & 4 \\
\hline $\begin{array}{l}\text { Instance } 1 \\
\text { mOFQS } \\
\alpha=0.9 \beta=0.1\end{array}$ & 14.9 & 23.9 & 16.3 \\
\hline $\begin{array}{l}\text { Instance } 2 \\
\text { mOFQS } \\
\alpha=0.1 \quad \beta=0.9\end{array}$ & 1.4 & 1.2 & 1.1 \\
\hline
\end{tabular}

Table 1: Paths values for the different metrics used 


\section{3 mOFQS metric}

In order to enable RPL to consider the remaining energy, the latency and the multiple instances beside the reliability using ETX, mOFQS includes the Power State $P S$, the delay $d$ of delivering a packet within two nodes in milliseconds and two parameters $\alpha$ and $\beta$. $\mathbf{m O F Q S ~ f o r m u l a ~ i s ~ s h o w n ~ b e l o w : ~}$

$$
m O F Q S=\frac{\alpha E T X * d}{P S^{\beta}}
$$

where $\alpha$ and $\beta$ are two tunable parameters with $\alpha=1-\beta$, $0<\alpha<1$ and $0<\beta<1$.

$\mathbf{m O F Q S}$ is an additive metric whose values over the path is the sum of the values at each hop. Each node chooses the path upward in its DODAG with the smallest value provided by mOFQS. First of all, varying $\alpha$ and $\beta$ allow us to differentiate between instances depending on their criticality level.

The idea is to multiply $\boldsymbol{E T X}$ by $d$ for every hop to get the links reliability while considering the delay of the packet delivery, then multiply the factor $E T X^{*} d$ by $\alpha$ to foster link quality and end-to-end delay for critical applications by increasing $\alpha . \alpha\left(E T X^{*} d\right)$ is then divided by $P S$ power $\beta$. Increasing or decreasing $\beta$ will similarly foster $P S$. If the application is critical, $\beta$ should be decreased (resp. $\alpha$ increased). For delay tolerant applications, increasing $\beta$ will result in a longer route while conserving the nodes power since the metric will weight more node energy level rather than link quality or end-to-end delay. Dividing $\alpha\left(E T X^{*} d\right)$ by $P S^{\beta}$ aims to foster routes where the nodes consumed less their batteries or are main powered. For one application, we favor $\alpha$ or $\beta$ against the other, and since $\alpha+\beta=1$, when one parameter increases the other decreases and vice-versa.

Figure 1 depicts a small network of 6 nodes running RPL, considering two different applications: one is critical and belongs to Instance 1 and the other is regular and belongs to Instance 2. Node 6 sends a packet to node 1 , available paths are: path 1: $6 \rightarrow 5 \rightarrow 2 \rightarrow 1$ or path $2: 6 \rightarrow 4 \rightarrow 3 \rightarrow 1$ or path $3: 6 \rightarrow 4 \rightarrow 3 \rightarrow 2 \rightarrow 1$. Table 1 shows the different paths metric values with $\boldsymbol{E T X}, \boldsymbol{H C}$ and $\boldsymbol{m O F Q S}$. For ETX alone, path 1 is the optimal one since it is the only metric used. For $\boldsymbol{E T X} \& \boldsymbol{H C}, \boldsymbol{E T X}$ is used for the critical traffic (Instance 1) and $\boldsymbol{H C}$ for the regular one (Instance 2), as we can see Instance 2 optimal path will be 1 or 2 since they count less hops, and for Instance 1, it will be path 1 which has $\boldsymbol{E T X}=7.5$. Neither $\boldsymbol{E T X}$ or $\boldsymbol{H C}$ take energy consumption and delay into consideration, unlike mOFQS where $\alpha$ and $\beta$ values will foster one path over the other. With mOFQS, in Instance 1 with critical traffic which requires minimal latency, we have to route the packets as fast as possible while guarantying a reliable link. Thus, we increment $\alpha(\alpha=0.9)$ fostering $E T X^{*} d$ (reliability and latency), which means decreasing $\beta(\beta=0.1)$. mOFQS fosters path 1 since it has better $\boldsymbol{E T X}$ and $d$ values than paths 2 and 3 . In Instance 2 , where the traffic is not critical, we increment $\beta$ $(\beta=0.9)$. Thus, we foster $P S$, which means that we might pass by a longer and less reliable route, while guaranteeing load balancing. Consequently forcing paths where nodes consumed less their batteries (path 3 where node 3 and 4 have more than $80 \%$ energy left in their batteries unlike path 1 where nodes 2 and 5 have less than $30 \%$ energy left). We achieve then a traffic distribution along the nodes by passing by path 3 and extending the network's lifetime.

\begin{tabular}{|c|c|c|}
\hline Data traffic & $\begin{array}{l}\text { Maximum } \\
\text { allowed delay }\end{array}$ & Reliability \\
\hline $\begin{array}{l}\text { DA - Data related to } \\
\text { the protection of the } \\
\text { distribution network }\end{array}$ & $<3 \mathrm{~s}$ & $>99.5 \%$ \\
\hline $\begin{array}{l}\text { DERs (Distributed Energy } \\
\text { Resources) - Data related } \\
\text { to the protection of the } \\
\text { distribution network }\end{array}$ & $<4 \mathrm{~s}$ & $<99.5 \%$ \\
\hline $\begin{array}{l}\text { Critical traffic of: DA, } \\
\text { DSM, AMI, DERs }\end{array}$ & $<5 \mathrm{~s}$ & $>99.5 \%$ \\
\hline Electric transport & $<10 \mathrm{~s}$ & $>98 \%$ \\
\hline $\begin{array}{l}\text { Non critical traffic of: } \\
\text { DSM \& AMI }\end{array}$ & $<15 \mathrm{~s}$ & $>98 \%$ \\
\hline $\begin{array}{l}\text { Non critical traffic of: } \\
\text { DA \& AMI }\end{array}$ & $<30 \mathrm{~s}$ & $>98 \%$ \\
\hline $\begin{array}{l}\text { Network configuration } \\
\text { traffic, normal AMI traffic }\end{array}$ & $<5 \min$ & $>98 \%$ \\
\hline Normal AMI traffic & $<4 \mathrm{~h}$ & $>98 \%$ \\
\hline $\begin{array}{l}\text { Network configuration } \\
\text { traffic }\end{array}$ & $<$ HoursDays & $>98 \%$ \\
\hline
\end{tabular}

Table 2: NAN requirements in terms of reliability

\subsection{Instances classification}

Traffic classes in SG are not yet standardized. In this paper, we use the classification presented in [24] for the requirements in terms of delay and reliability in a Neighborhood Area Network (NAN) as shown on Table 2. The aforementioned classification sorts the traffic into 9 different classes, ranging from delays inferior than 3 seconds with reliability $>99.5 \%$ for the most critical class to delays of hours/days with a reliability of $>98 \%$ for the least critical class. In our model, we have gathered these 9 classes into 3 classes with 3 main instances:

- Instance 1: critical traffic with an authorized delay ranging between 1 and 30 seconds and a reliability of $>99.5 \%$ packets received with $\alpha=0.9$ and $\beta=0.1$

- Instance 2: non-critical traffic with an authorized delay of days and a reliability of $>98 \%$ packets received with $\alpha=0.1$ and $\beta=0.9$

- Instance 3: periodic traffic with an authorized delay ranging between 5 minutes and 4 hours and a reliability of $>98 \%$ packets received with $\alpha=0.3$ and $\beta=0.7$

In this classification, we increment $\alpha$ for the critical traffic thus fostering the link quality and end to end delay assured by $\boldsymbol{E T X}$ and $d$, which results in routing the packets in a reliable and faster path. For less critical traffic we increment 
$\beta$ which leads to fostering paths where the nodes consumed less their batteries and then achieving a load balancing. We note that our model is not limited to this classification and for any other one $\alpha$ and $\beta$ can be modified depending on the network characteristics.

\section{SIMULATION SETUP}

\begin{tabular}{ll} 
Parameters & Values \\
\hline OS & Contiki master version \\
\hline Simulator & $\begin{array}{l}\text { Cooja; Radio Model: } \\
\text { Unit Disk Graph Medium }\end{array}$ \\
\hline $\begin{array}{l}\text { Communication } \\
\text { protocols }\end{array}$ & $\begin{array}{l}\text { CSMA, RDC contikimac, } \\
\text { IEEE 802.15.4, channel 26, } \\
\text { ContikiRPL, IPv6 }\end{array}$ \\
\hline OF & $\begin{array}{l}\text { 1- OFQS with 2 instances } \\
\text { 2- MRHOF(ETX) \& OF0(HC) }\end{array}$ \\
\hline Number of nodes & 35 clients and 1 server \\
\hline Deployment area & 200m x 200m \\
\hline Transmission & $50 \mathrm{~m}$ \\
Interference range & \\
\hline Transmission & 100\% \\
\hline Reception ratio & Zolertia Z1 \\
\hline Sensors & $30 \mathrm{~kb}$ \\
\hline Packet size & 1 packet every 3 to 4 minutes \\
\hline Sending interval & \\
\hline
\end{tabular}

Table 3: Parameters of the simulation

In order to evaluate our appraoach, simulation were performed on Contiki OS [13] using Cooja simulator alongside with its emulator MSPSIM. MPSIM provides accurate emulation at both cycle-level for the MSP430 micro-controller and bit-level for the CC2420 radio transceiver, which will allow accurate energy estimation. Simulation parameters are presented in Table 3. Zolertia Z1 motes were emulated, we used the Energest module in Contiki to estimate the battery levels by extracting the values for the energy consumption from the $\mathrm{Z} 1$ datasheet $^{2}$. Motes were given two different battery levels at bootstrap (10 nodes have $60 \%$ of energy of the others) in order to highlight the energy consumption. The topology consists of 35 client nodes randomly positioned that send UDP packets to the server placed in the middle, every 3 to 4 minutes. We considered a $100 \%$ transmission/reception ratio. We are aware that this is not a so realistic setting but this allows a fair comparison of $\boldsymbol{E T X}, \boldsymbol{H C}$ and our metric under the best case scenario, which is from our perspective, what we aim to. As multiple RPL instances are not fully implemented

\footnotetext{
$\overline{{ }^{2} \text { http://zolertia.sourceforge.net }}$
}

in Contiki, we used an implementation ${ }^{3}$ where multiple instances are supported and adapted it. Only upward traffic was considered. $O F Q S$ with two instances: critical and non critical (Instance 1 and Instance 2 resp.) was compared to RPL with two instances : MRHOF with ETX metric for critical traffic and $O F O$ with $\boldsymbol{H C}$ metric for less critical traffic. For the rest of the paper, we will indicate $O F Q S$ for $O F Q S$ with the two instances mentioned above, and $M R H O F / O F 0$ for RPL with $\boldsymbol{E T X}$ and $\boldsymbol{H C}$. Tests were repeated 10 times and the simulation stops when $20 \%$ of the nodes have consumed all their energy. All simulation results are measured with a $95 \%$ of confidence interval.

\section{PERFORMANCE EVALUATION}

\subsection{Network lifetime and load balancing}

Figure 2 shows the percentage of nodes that did not exhaust their batteries during the simulation. We can see that for MRHOF / OF0 starting hour 21 battery nodes start to drain and within 9 hours (after 30 hours), 20\% of the nodes exhausted their batteries, which makes the simulation stop as previously defined. For $O F Q S$, and for the first 38 hours all nodes are still functional and none of them has consumed its total energy. Starting $39^{\text {th }}$ hour, the nodes batteries start to drain and the network stops after 51 hours. OFQS presents a gain of 21 hours, which means around $59 \%$ on the network lifetime compared to $M R H O F / O F O$, this is due to the $P S$ factor, which after a certain period of time and when the nodes start to consume their batteries, foster the switch to other routes with better battery nodes. In the same way, we can see in Figure 3 that after 24 hours of operation, 57.1\% of the nodes have still $70 \%$ of their energy with $O F Q S$ compared to $8.6 \%$ with $M R H O F / O F 0$. Besides that, $5.7 \%$ have $20 \%$ and $10 \%$ left energy with $M R H O F / O F 0$ compared to $0 \%$ of the nodes with OFQS. Finally after 24 hours $8.6 \%$ of the nodes consumed their total energy with $M R H O F / O F 0$ compared to $0 \%$ with $O F Q S$. This is mainly due also to the $P S$ factor which switches into nodes that consumed less their batteries achieving then a better load balancing of traffic among the nodes.

\subsection{End-to-End delay}

Figure 4 shows the variation of End-to-End delay (in $\mathrm{ms}$ ) for both $O F Q S$ and $M R H O F / O F 0$ within simulation time. We can see that End-to-End delay with $O F Q S$ is always below $M R H O F / O F 0$. Even though that $\boldsymbol{H C}$ favors paths with fewer hops, these paths are generally longer with potential poorer connectivity. On the other hand, ETX is not also aware of the delays due to interference on the links and queuing in the nodes as long as the packets are transmitted; therefore, sending a packet with less retransmissions does not mean sending it on a faster link. OFQS chooses faster routes due to the $d$ factor in $\mathbf{m O F Q S ~ t h a t ~ t a k e s ~ t h e ~ d e l a y ~ b e t w e e n ~ t w o ~}$ hops into consideration, which will foster faster links with less interference and congestion that $\boldsymbol{E T X}$ and $\boldsymbol{H C}$ are not

\footnotetext{
$\overline{{ }^{3} \text { https://github.com/jeremydub/contiki }}$
} 


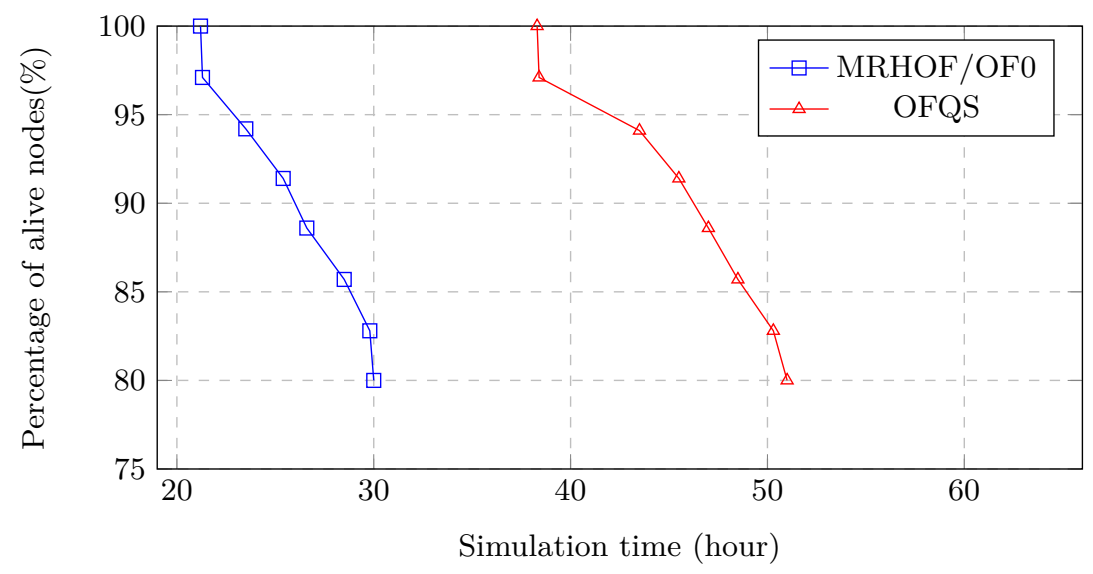

Figure 2: Network lifetime variation

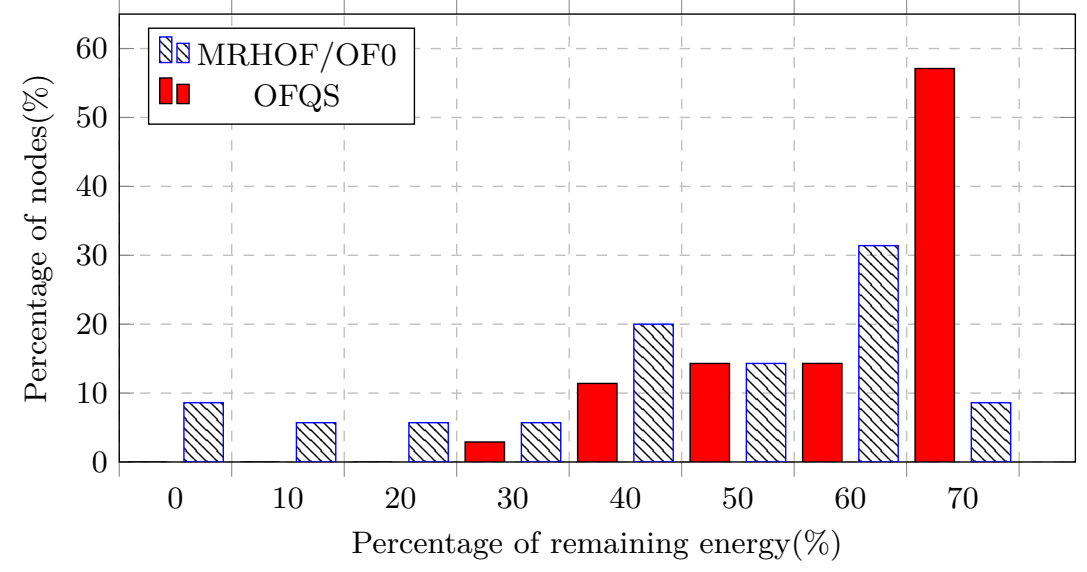

Figure 3: Remaining energy distribution among the nodes after 24 hours

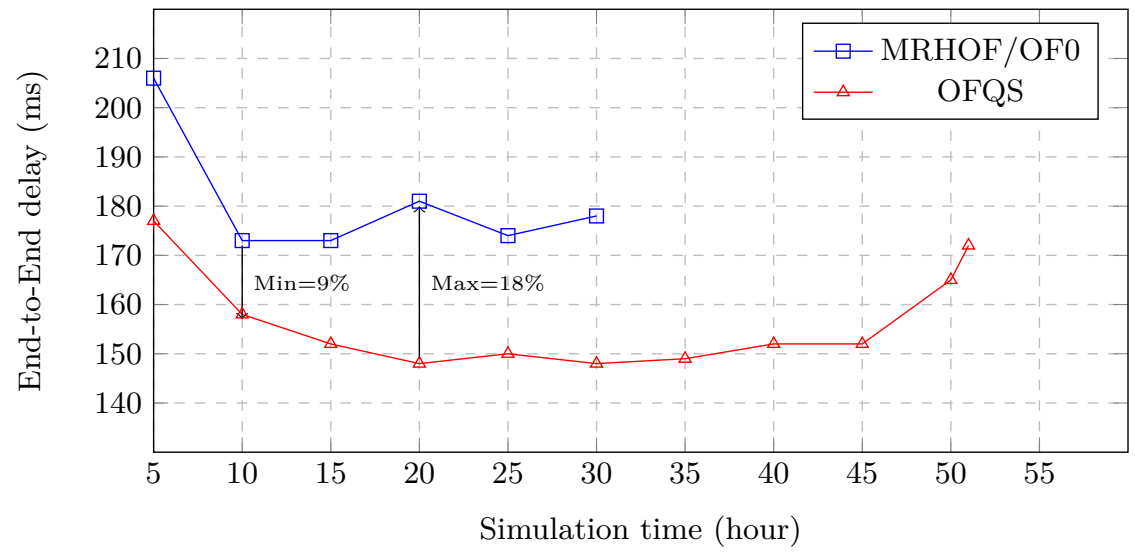

Figure 4: End-to-End delay variation with time

aware of. Beside that, we can see that the End-to-End delay decreases up to the first 20 hours. This is due to the fact that the battery nodes were still full and such none of them is in a critical state. That's why the $d$ factor favors faster routes.
After that, between 20 and 45 hours, the delay is mostly stable which is due to the variation of the battery levels which is affecting choosing faster routes. Finally after 45 hours and up to the end of the simulation, we can see an increase in the 


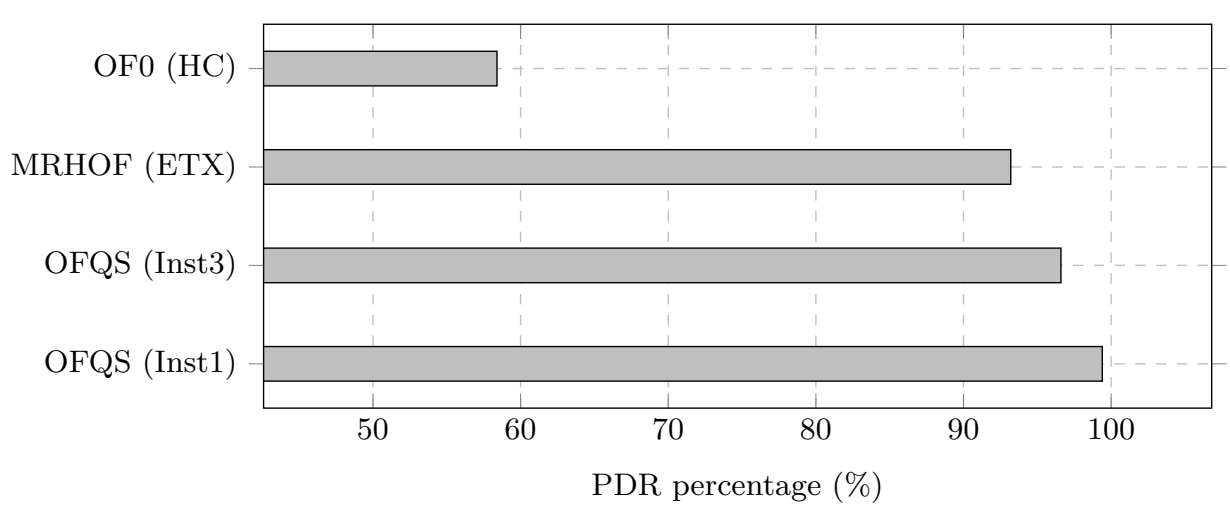

Figure 5: Packet delivery ratio percentage for the different OFs/metrics

End-to-End delay which is mainly due to the depletion of certain battery nodes which leads to choosing longer routes to maximize the network lifetime. OFQS average End-to-End delay during the simulation makes an improvement between $9 \%$ and $18 \%$ compared to $M R H O F / O F O$, and stays within the time requirements limits previously defined in section 4.4 .

\subsection{Packet delivery ratio}

Figure 5 shows the PDR percentage for the different metrics used. We can see that for $\boldsymbol{H C}$, the PDR is less than $60 \%$. This is due to the route selection in this metric that only relies on the number of hops from the sink without any reliability mechanism. For $\boldsymbol{E T X}$ the PDR is $93.2 \%$ compared to $99.4 \%$ for $\mathbf{m O F Q S}$ with Instance $1(\alpha=0.9 \& \beta=0.1)$ and $96.6 \%$ for Instance $3(\alpha=0.1 \& \beta=0.9)$, which shows that $O F Q S$ overpasses both $\boldsymbol{E T X}$ and $\boldsymbol{H C}$ in terms of reliability. mOFQS considers besides $\boldsymbol{E T X}$, the delay of sending a packet in one hop which reflects the interference delay on that hop, allowing more reliable routes to be chosen by multiplying those two factors $(\boldsymbol{E T X} \& \boldsymbol{d})$. Finally, we note that the achieved PDR percentages with $O F Q S$ are relatively close to the SG requirements that were defined in Section 4.4.

\section{CONCLUSION AND FUTURE WORK}

In this paper, we propose $O F Q S$, a standard-compliant objective function with a multi-objective metric mOFQS that considers by design the delay, the remaining energy and the quality of the links in order to be used with the standard protocol RPL in a SG environment. OFQS dynamically adapts the routing to the number of instances in a network providing a differentiation based on the requirements of the SG applications. Simulation results show that OFQS achieves significant improvement in terms of End-to-End delay, network lifetime and PDR while insuring a load balancing among the nodes, compared to $M R H O F$ with $\mathbf{E T X}$ and $O F O$ with $\boldsymbol{H C}$, in a multiple instances environment. As future work, we will test $O F Q S$ on a real testbed and a large scale smart grid network (SoMel SoConnected project) to assess its performance in a real case scenario. Furthermore, we want to explore how to improve downward and point to point routing in RPL in order to fit with the requirements of the SG and propose interoperability solutions on how RPL can coexist with other existing standards.

\section{ACKNOWLEDGMENTS}

This work was partially funded by a grant from the MEL (Métropole Européenne de Lille), SoMel SoConnected project (Ademe, PIA2), Yncréa Haut-de-France and CPER Data.
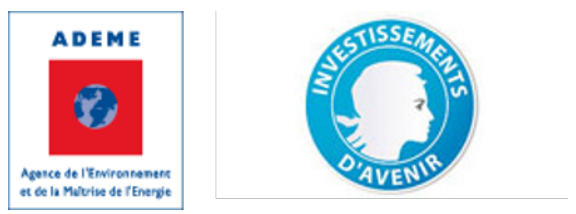

\section{REFERENCES}

[1] Emilio Ancillotti, Raffaele Bruno, and Marco Conti. "RPL Routing Protocol in Advanced Metering Infrastructures: an Analysis of the Unreliability Problems". In: Sustainable Internet and ICT for Sustainability (SustainIT). IEEE. 2012, pp. 1-10.

[2] Emilio Ancillotti, Raffaele Bruno, and Marco Conti. "The role of the RPL routing protocol for smart grid communications". In: Communications Magazine, IEEE 51.1 (2013), pp. 75-83.

[3] Mai Banh et al. "Performance evaluation of multiple RPL routing tree instances for Internet of Things applications". In: Advanced Technologies for Communications (ATC), International Conference on. IEEE. 2015, pp. 206-211.

[4] BKW. Smart Grid Taxonomy. A system view from a grid operator's perspective. 2015. URL: http://www. bkw . ch / fileadmin / user_ upload / 3 _ Gemeinden _ EVU/gem_smart_grid_systematik_en.pdf.

[5] Faycal Bouhafs, Michael Mackay, and Madjid Merabti. "Links to the future: communication requirements and challenges in the smart grid". In: IEEE Power and Energy Magazine 10.1 (2012), pp. 24-32. 
[6] Kenneth C Budka et al. "Communication network architecture and design principles for smart grids". In: Bell Labs Technical Journal 15.2 (2010), pp. 205-227.

[7] Nancy Cam-Winget, Jonathan Hui, and Daniel Popa. Applicability Statement for the Routing Protocol for Low Power and Lossy Networks (RPL) in AMI Networks. Internet-Draft. draft-ietf-roll-applicability-ami13. IETF, Apr. 2016.

[8] Silvia Capone et al. "An Energy Efficient and Reliable Composite Metric for RPL Organized Networks". In: International Conf. on Embedded and Ubiquitous Computing (EUC). IEEE. 2014.

[9] Brandon Davito, Humayun Tai, and Robert Uhlaner. "The smart grid and the promise of demand-side management". In: McKinsey on Smart Grid 3 (2010), pp. 844.

[10] Piergiuseppe Di Marco et al. "MAC-aware routing metrics for low power and lossy networks". In: INFOCOM, Proceedings. IEEE. 2013.

[11] Richard Draves, Jitendra Padhye, and Brian Zill. "Comparison of routing metrics for static multi-hop wireless networks". In: ACM SIGCOMM Computer Communication Review 34.4 (2004), pp. 133-144.

[12] Richard Draves, Jitendra Padhye, and Brian Zill. "Routing in multi-radio, multi-hop wireless mesh networks". In: Proceedings of the 10th annual international conference on Mobile computing and networking. ACM. 2004, pp. $114-128$.

[13] Adam Dunkels, Bjorn Gronvall, and Thiemo Voigt. "Contiki-a lightweight and flexible operating system for tiny networked sensors". In: Inter. Conf. on Local Computer Networks (LCN). IEEE. 2004.

[14] Olfa Gaddour et al. "OF-FL: QoS-aware fuzzy logic objective function for the RPL routing protocol". In: International Symposium on Modeling and Optimization in Mobile, Ad Hoc, and Wireless Networks (WiOpt). IEEE. 2014.

[15] Omprakash Gnawali and Philip Levis. The Minimum Rank with Hysteresis Objective Function. RFC 6719. RFC Editor, 2012.

[16] Vehbi C Gungor, Bin Lu, and Gerhard P Hancke. "Opportunities and challenges of wireless sensor networks in smart grid". In: IEEE Transactions on Industrial Electronics 57.10 (2010), pp. 3557-3564.

[17] Patrick Olivier Kamgueu et al. Energy-based routing metric for RPL. Research Report. Inria, 2013.

[18] Patrick-Olivier Kamgueu, Emmanuel Nataf, and Thomas Ndie Djotio. "On design and deployment of fuzzy-based metric for routing in low-power and lossy networks". In: Local Computer Networks Conference Workshops (LCN Workshops). IEEE. 2015.

[19] Panagiotis Karkazis et al. "Design of primary and composite routing metrics for RPL-compliant wireless sensor networks". In: Int. Conf. on Telecommunications and Multimedia (TEMU). IEEE. 2012.
[20] Hyung-Sin Kim, Jeongyeup Paek, and Saewoong Bahk. "QU-RPL: Queue utilization based RPL for load balancing in large scale industrial applications". In: 12th International Conference on Sensing, Communication, and Networking (SECON). IEEE. 2015.

[21] Nguyen Thanh Long et al. "QoS-aware cross-layer mechanism for multiple instances RPL". In: International Conference on Advanced Technologies for Communications (ATC). IEEE. 2013, pp. 44-49.

[22] Jovan Radak, Nathalie Mitton, and David Simplot-Ryl. "Using Battery Level as Metric for Graph Planarization". In: International Conference on Ad Hoc Networks and Wireless (AdHocNow). 2011, pp. 58-71.

[23] Gowdemy Rajalingham et al. "Quality of service differentiation for smart grid neighbor area networks through multiple RPL instances". In: Proc. symposium on QoS and security for wireless and mobile networks. ACM. 2014, pp. 17-24.

[24] Mouna Rekik. "Routage géographique multi-chemin basé sur l'intelligence d'essaim pour réseaux de capteurs et d'actionneurs sans fil : application aux Smart Grids". PhD thesis. Univ. Lille 1, 2016.

[25] Nermin Suljanovic et al. "Requirements for communication infrastructure in smart grids". In: Energy Conference (ENERGYCON). IEEE. 2014.

[26] Pascal Thubert. Objective Function Zero for the Routing Protocol for Low-Power and Lossy Networks (RPL). RFC 6552. 2012.

[27] Jean-Philippe Vasseur et al. Routing metrics used for path calculation in low-power and lossy networks. InternetDraft. draft-ietf-roll-routing-metrics-19. IETF, 2012.

[28] Di Wang et al. "RPL-based routing for advanced metering infrastructure in smart grid". In: Int. Conf. on Communications Workshops (ICC). IEEE. 2010.

[29] Tim Winter, Pascal Thuber, Anders Brandt, et al. RPL: IPv6 Routing Protocol for Low-Power and Lossy Networks. RFC 6550. RFC Editor, 2012.

[30] Sooyeol Yang et al. "A routing metric for load balance in wireless mesh networks". In: International Conference on Advanced Communication Technology. (ICACT). IEEE. 2009, pp. 1560-1565. 